\title{
Surgical Site Infections in Developing Countries: Current Burden and Future Challenges
}

\author{
Ahmed Morad Asaad ${ }^{1 *}$ and Samir Ahmad Badr ${ }^{2}$ \\ ${ }^{1}$ Department of Microbiology, College of Medicine, NajranUniversity, Najran, Saudi Arabia \\ ${ }^{2}$ Department of Surgery, Banha Teaching Hospital, General Organization for Teaching Hospitals \& Institutes (GOTHI), Egypt
}

*Corresponding author: Ahmed Morad Asaad, Coordinator of Microbiology Department, College of Medicine, Najran University, P.O Box 1988, Najran, Saudi Arabia, Tel: +966-175428516; Fax: +966-175442419; E-mail: ahmedmoradasaad@hotmail.com

Received date: November 21, 2016; Accepted date: November 24, 2016; Published date: November 30, 2016

Copyright: $\odot 2016$ Asaad AM, et al. This is an open-access article distributed under the terms of the Creative Commons Attribution License, which permits unrestricted use, distribution, and reproduction in any medium, provided the original author and source are credited.

\section{Editorial}

Despite the great advance in surgical techniques, antimicrobial prophylaxis, environmental improvements in operating rooms, and effective infection prevention strategies, surgical site infections (SSIs) remain one of the most common causes of health care associated infections (HAIs), ranking first, when asymptomatic urinary tract infection is not taken into account. The SSIs causes increased morbidity and mortality, ranging from wound discharge associated with superficial skin infection to life-threatening conditions such as severe sepsis. The SSIs are responsible for an increased economic burden to healthcare systems in terms of additional postoperative hospital duration and costs $[1,2]$.

Over the past few decades, national and international guidelines and standards have been established and regularly updated in several developed countries to reduce SSIs rates. In high-income countries, effective control of SSIs depends on a wide scope of interventions that includes surveillance, healthcare-bundles, antimicrobial prophylaxis, eradication of carrier status, infection control programs and education $[3,4]$.

Globally, SSIs rates have been reported to range from $2.5 \%$ to $41.9 \%$ with significantly higher rates in developing countries. Besides, the surgical mortality in developing nations is 10 times higher than developed countries and deaths attributed to anaesthesia are 1000-fold higher [1]. In a recent meta-analysis report of 220 international studies investigating SSIs rates in developing countries, the cumulative incidence ranged from 0.4 to 30.9 per 100 patients and from 1.2 to 23.6 per 100 surgical procedures, while the pooled cumulative incidence was 11.8 per 100 patients [2]. Conversely, in a nationwide study undertaken in the USA, the cumulative incidence of SSIs was 2.6 per 100 surgical procedures [5]; 2.9 per 100 surgical procedures in different European countries [6], and 1.6 per 100 procedures in Germany [7].

Within the scope of developing countries, the higher SSIs rate is not surprising and may clearly reflect the typical situation of limitedresource hospitals in these countries as a whole. Several reasons have been proposed to explain this fact. The cornerstone is the lack of adherence to recommended infection control guidelines and suboptimal practices in many developing countries. Meanwhile, the hospitals IC committees in several countries focus just on outbreaks of hospital infections without committee discussion of surveillance or endemic HAI $[2,3,8]$. A major problem faced by health care professionals in developing countries is the lack of microbiology laboratory capacity at the hospitals with subsequent scarcity and frequent unreliability of microbiological data and other diagnostic procedures [2,9]. Another important issue is the absence or limited written policies or guidelines on proper perioperative antibiotic policies. This problem is more worsened in developing countries, where there is antibiotic misuse and abuse [10]. Sterilization and disinfection of surgical and other medical equipment is another area of concern, which might be due to a lack of defined policies and procedures in many hospitals, or improper instrument sterilization and disinfection techniques in other health care facilities [3,4]. Other significant problems include absence of effective surveillance and educational programs, multidisciplinary quality improvement teams, careful monitoring and feedback, and a mandatory hand hygiene practice [3].

In conclusion, the SSIs in developing countries are on the rise associated with a dramatic increase in patients morbidity, and mortality. Much effort is required from health decision makers, surgeons and all health care workers in hospitals from developing countries to combat SSIs burden. Adherence to strict infection control guidelines, sterilization and disinfection policies and procedures, powerful microbiology laboratories, efficient surveillance programs with active feedback to surgeons, and proper antimicrobial stewardship, seem to be crucial to better control SSIs rates in developing countries.

\section{References}

1. World Health Organization. Report on the burden of endemic health careassociated infection worldwide: clean care is safer care. World Health Organization website. 2016.

2. Allegranzi B, Bagheri Nejad S, Combescure C, Graafmans W, Attar H, et al. (2010) Burden of endemic health-care-associated infection in developing countries: systematic review and meta-analysis. Lancet 377: 228-241.

3. Anderson DJ, Podgorny K, Berrios-Torres SI, Bratzler DW, Dellinger EP, et al. (2014) Strategies to prevent surgical site infections in acute care hospitals: 2014 update. Infect Control Hosp Epidemiol 35: 66-88.

4. Centers for Disease Control and Prevention. Procedure-associated module: SSI. 2016.

5. Gaynes RP, Culver DH, Horan TC, Edwards JR, Richards C, et al. (2001) Surgical site infection (SSI) rates in the United States, 1992-1998: the National Nosocomial Infections Surveillance System basic SSI risk index. Clin Infect Dis 33: 69-77.

6. HELICS (2016) Surveillance of surgical site infections: SSI statistical reportsurgical site infections 2004. March, 2006.

7. Gastmeier P, Geffers C, Brandt C, Zuschneid I, Sohr D, et al. (2006) Effectiveness of a nationwide nosocomial infection surveillance system for reducing nosocomial infections. J Hosp Infect 64: 16-22.

8. Weinshel K, Dramowski A, Hajdu A, Jacob S, Khanal B, et al. (2015) Gap Analysis of Infection Control Practices in Low- and Middle-Income Countries. Infect Control Hosp Epidemiol 36: 1208-1214. 
Citation: Asaad AM, Badr SA (2016) Surgical Site Infections in Developing Countries: Current Burden and Future Challenges. Clin Microbiol 5: e136. doi:10.4172/2327-5073.1000e136

Page 2 of 2

9. Dramowski A (2014) Infection Prevention and Control: A Guide for Healthcare Workers in Low-Resource Settings. Cape Town: Bettercare, 2014.
10. Okeke IN, Laxminarayan R, Bhutta ZA, Duse AG, Jenkins P, et al. (2005) Antimicrobial resistance in developing countries, I: recent trends and current status. Lancet Infect Dis 5: 481-493. 